\title{
Simulation of Pulsed Laser Material Processing Controlled by an Extended Self-Organizing Kohonen Feature Map
}

\author{
Gábor J. Tóth ${ }^{1}$, Tamás Szakács and András Lörincz
}

Institute of Isotopes, P.O.B. 77, Budapest, Hungary H-1525,

1 Eötvös Loránd University, Budapest, Hungary

E-mails: jtoth@obelix.iki.kfki.hu, lorincz@obelix.iki.kfki.hu

We have simulated the control of laser material processing. The process to control was surface polishing and milling. In the simulations we assumed pulsed ultraviolet laser beams of 100 nanosecond duration. The controller was an artificial neural network (ANN): an extended self-organizing Kohonen feature map. The controller was trained - tuned - on examples of laser evaporation of one dimensional 'surfaces' by using a Widrow-Hoff type Delta-rule for error correction. The strength of the approach lies in the speed of parallel computing and the adaptive properties of the controller in a changing environment, like drift in laser properties, optical components, etc. Results show more than an order of magnitude improvement in surface roughness after an ANN designed laser shot onto a large surface. Restrictions of the model are discussed.
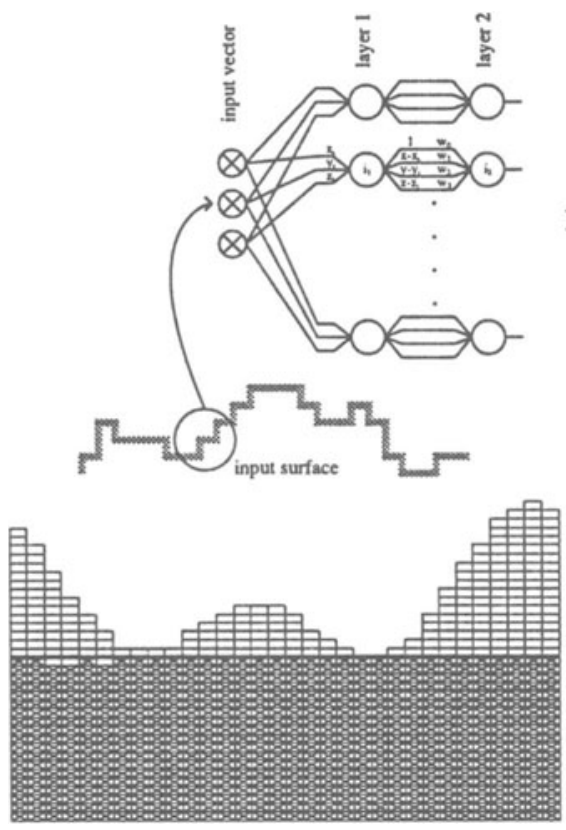

Figure 2: Smoothing surfaces: typical performance of the trained network. Empty boxes correspond to the original surface, filled boxes to surface after the shot. Wide line shows the target surface level. Box size is $200 \mathrm{~nm}$ horizontally and $100 \mathrm{~nm}$ vertically, material is aluminium.
Figure 1: The architecture of the network

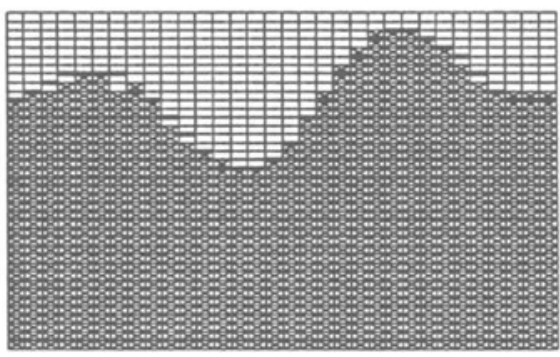

Figure 3: Milling patterns: typical performance of the trained network. Empty boxes correspond to the original surface, filled boxes to surface after the shot. Wide line shows the target surface level. Box size is $200 \mathrm{~nm}$ horizontally and $100 \mathrm{~nm}$ vertically, material is aluminium. 